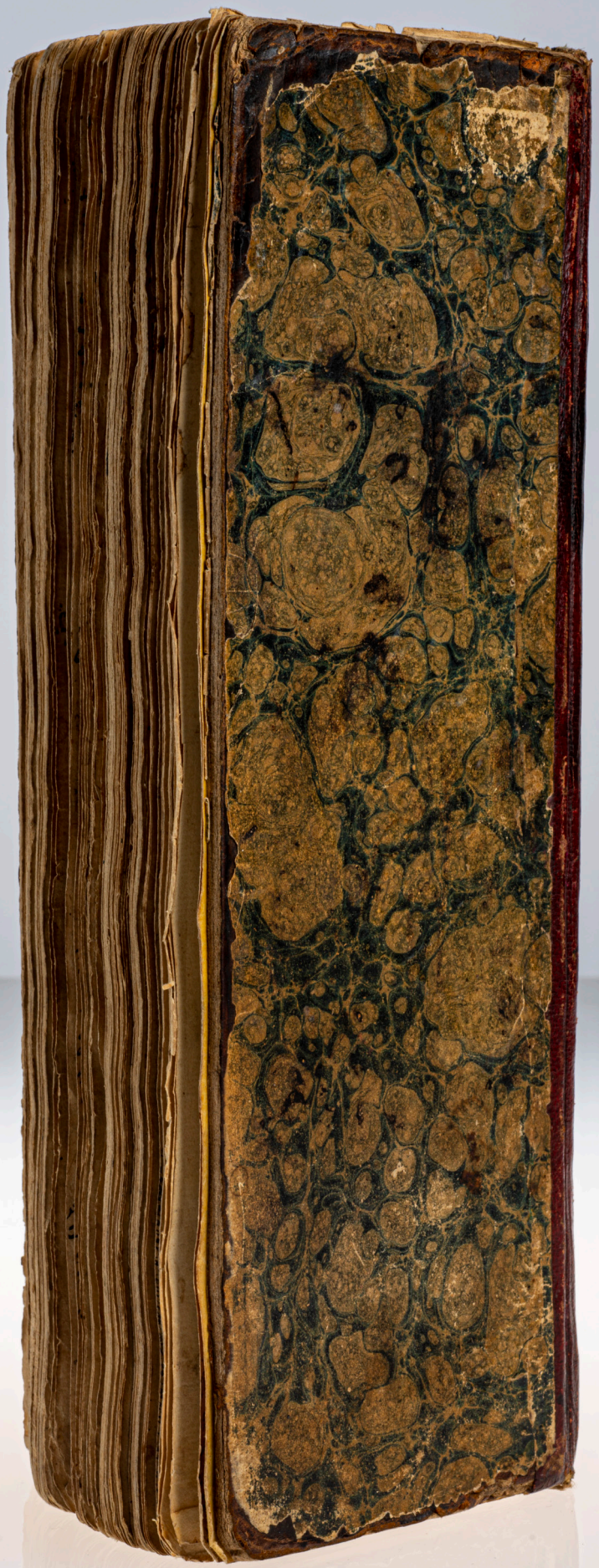




\section{A Radical Discourse of Desire: Küsnâme}

\section{Selim S. Kuru*}

Found in the Suna and İnan Kıraç Foundation Manuscript Collection within the Istanbul Research Institute, under the title "Name-i Tacîzâde Çelebi rahmetu'l-lahu 'aleyhi," this short work, which was preserved in a miscellaneous collection [тестиа] and kept for a few generations in the Baldırzâde family, ${ }^{1}$ is thought to be Küsnâme ("Expression of Desire"), the work of Tacîzâde Cafer Çelebi (d. 1515), a prominent public servant and poet. ${ }^{2}$

The Ottoman literary biographer Âşık Çelebi (d. 1572) mentions a work by Tâcîzâde that he labels a satirical work (hezl), with a title that may be read as Küsnâme and without any additional commentary. Yet, even the mention of the text implies its popularity. In addition to his works in Ottoman Turkish, Tâcîzâde Cafer Çelebi was famous for his verse and prose in the Persian language-even though his Persian was criticized by his friend Müeyyedzâde (d.1516) as being "Üngüri” (i.e., "Ankara Persian”). Working as the head of the Ottoman chancery under Bayezid II and later Selim I, Tâcîzâde would be executed upon Selim l's order for a reason yet to be clarified. He was famous for his romance Hevesnâme (1494), which versifies a realistic autobiographical love story between Cafer and a woman-unusual for romances of the late fifteenth century, and is told in high literary fashion. This work would establish itself for centuries as one of the sources for a fashionable genre in Ottoman-Turkish literature. Tâcîzâde, with his poetry collection, divan, and his Hevesnâme (Book of Desire), stands at the early stages of classical Ottoman literature as an innovative and famous poet and prose stylist. ${ }^{3}$

The only extant copy of Küsnâme consists of ninety-three rhyming couplets. In this work (not included in the poet's divan), Tâcizâde Cafer Çelebi describes his beloved, who had a mean but playful attitude, in traditional similes such as "tall as a cypress" and "with hair like hyacinths." He goes on to write about his suffering during their separation. The description of the physical features of his beloved and the suffering of the poet continues until couplet forty-five, after which Küsnâme presents an unexpected narrative turn as the poet expresses his carnal desire rather than his pure love:

You turned my tears into a fountain I should pull you aside and take you in my arms ${ }^{4}$

This is then followed by couplets expressing sexual desire. Except for satirical works, sexual acts in OttomanTurkish language and literature were mostly expressed in an obscure manner using a string of similes, but in "Küsnâme" the sexual act is described in detail; the lover openly expresses his desire to put his arm around his beloved's neck, tousle his hair, undo his sash, and take off his kaftan and shirt, thus finding a poetic form for such sexual acts that culminates from a description of foreplay into metaphors with direct reference to sexual intercourse.

I wish I could lead the ruby tipped arrow, into the silver box, oh my dear love $^{5}$

Küsnâme must have drawn concerned attention at the time due to this highly anomalous approach to love-hence Âşık Çelebi's consideration of it as satirical-for it is mentioned in the sources discussing Cafer Çelebi but never elaborated on. It was, however, hidden like satirical works of a similar vein and kept only in miscellaneous collections. As a matter of fact, Âşı Çelebi, after relating a conversation between the poet and the sultan, where the sultan condemns Tâcîzâde's poetry as "empty of the pleasures of love" ("eşârunuzdan çâşni-yi ışk madûmdur"), states, "This is the impact of love for women on him, for that reason his poetry lacks sweetness and its content is devoid of passion."

As an expression of the carnal desire of a poet rather than any realized act, Küsnâme is an early example that subverts high literary love discourse in Ottoman Turkish to the radical manner it realistically describes-the lover's sexual desire for his beloved and the sexual intercourse he seeks.
*University of Washington, selims@uw.edu

1 Mecmua-i Baldırzâde, Suna and İnan Kıraç Foundation Manuscript Collection, SVIKV, IAE ŞR 525/9, 140b-141b. The mecmua also includes fatwas from Ebussuud (d. 1574) and other Islamic jurists; waqf deeds, song, poems, prayers, translations of hadiths, Quranic suras, letters; Turkish, Arabic, Azerbaijani, and Persian notes and quotations; copies of imperial edicts; biographical notes on the members of Baldırzâde family, etc. For a full list of the contents, see Günay Kut et al., eds., Istanbul Araștırmalan Enstitüsü Yazma Eserler Kataloğu, v. 1 (İstanbul: İstanbul Araştırmaları Enstitüsü, 2014), 1346-1356. The manuscript is currently displayed at the Memories of Humankind: Stories from the Ottoman Manuscripts exhibition (October 18, 2019 - July 25, 2020) at the Istanbul Research Institute.

2 For a detailed introduction of this manuscript, see Fatma Büyükkarcı Yılmaz, "On yedinci yüzyıldan bir kırkambar: Baldırzâde ailesine ait bir mecmua," Turkish Studies 8, no. 1 (2013): 549-578. Along with descriptions and quotations from various works found in the miscellaneous collection, this article also includes a description of the "Nâme-i Tâcîzâde Cafer Çelebi" and suggests it as the "Küsnâme." The article also includes transcriptions of a selected 49 couplets from the work (p. 559-564).

3 Âşı Çelebi, Meşấïü'ş-şu'arâ, ed. Filiz Kılıç (İstanbul: İstanbul Araştırmaları Enstitüsü, 2010), 460-461.

4 Gözüm yaşını döndürdün bınara, koçaklasam seni çeksem kenara, 141a.

5 Gümüş sandukaya iy cân-ı cânân, revân itsem hadeng-i la'l-peykân, 141a.

6 Âşık Çelebi, Meşấirǚş-şu'arâ, 460. 
$|4|$

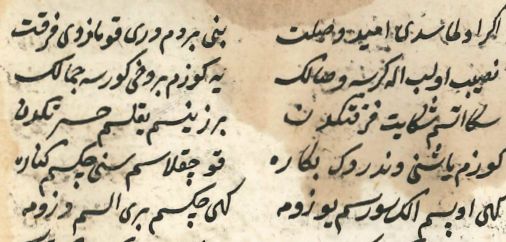

ون

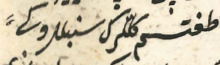

كاون

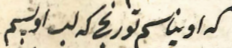

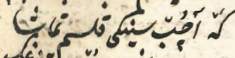

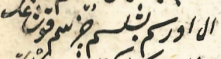

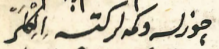

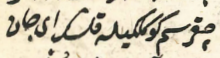

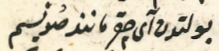

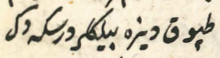

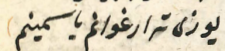

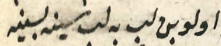

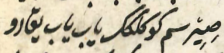

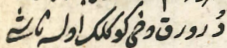

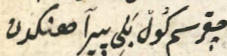

il this

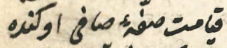

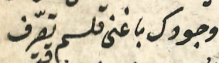

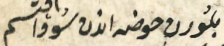

نوكنح

كlip

का

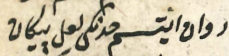

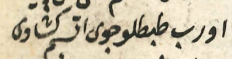

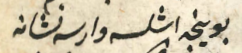

بز

ن :

(4) 6 क 6 o

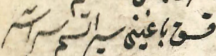

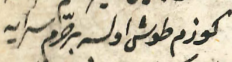

जcaitogdelus!

rbuil ilijingos

وروبواند

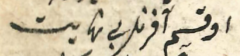

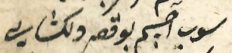

S1

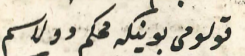

E

كز

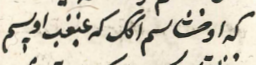

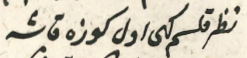

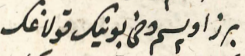

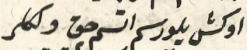

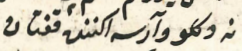

م6

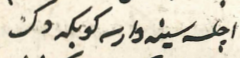

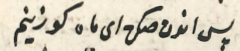

ק

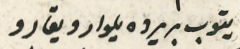

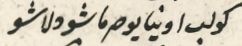

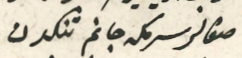

i

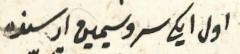

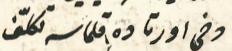

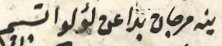

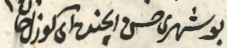

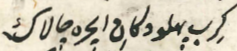

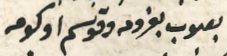

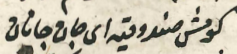

"و

"و

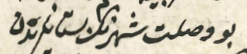

كُون

"6

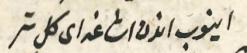

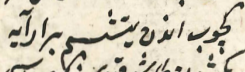

كTris

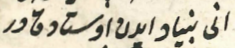

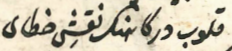

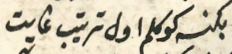

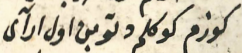

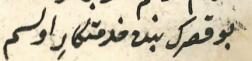

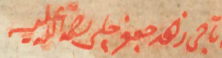

cosojioglstats.

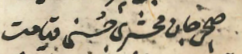

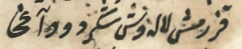

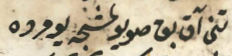

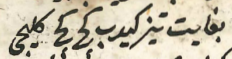

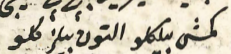

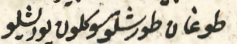

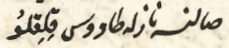

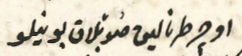

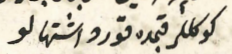

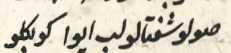

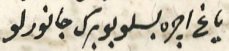

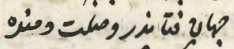

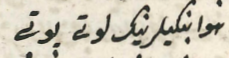

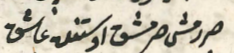
"

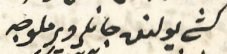
3

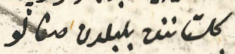

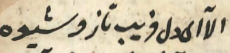

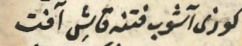
is ks Jic sigess

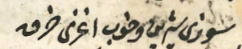

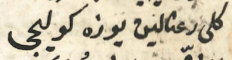

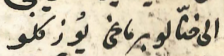

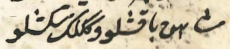

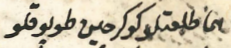

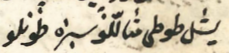
مار

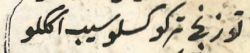

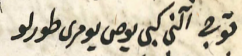
بان

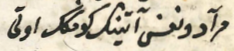

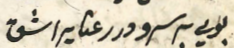

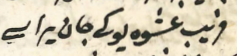

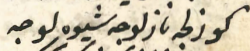

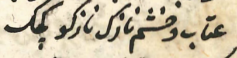

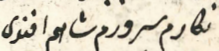

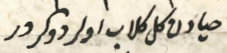

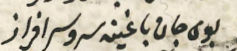

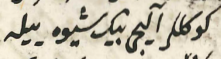

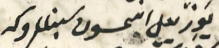
ته: كان مجك

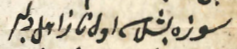

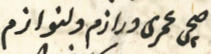

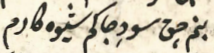
3"

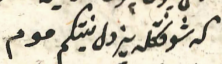

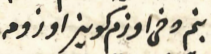

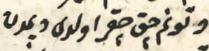

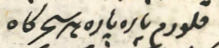
家 "و

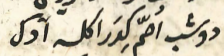

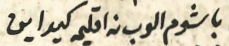

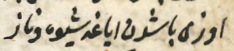
?

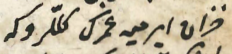

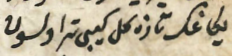

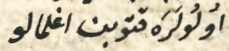
?

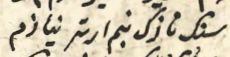
onts

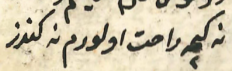

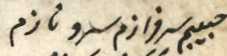

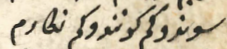

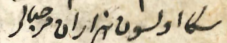
(2)

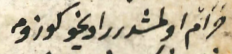
vis i $i$

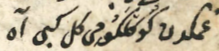
توك 3.5.

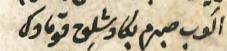

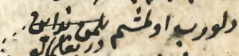

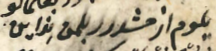

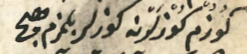

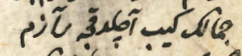
Sor 每

f1 


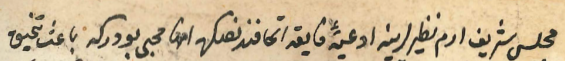
Co

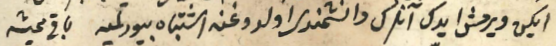

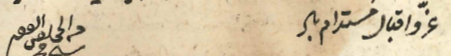

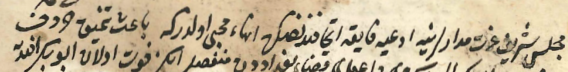

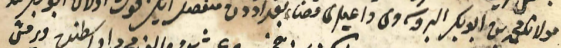

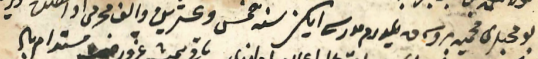

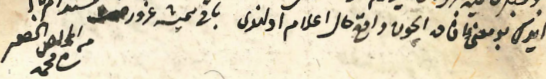

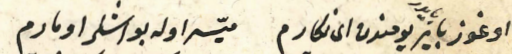

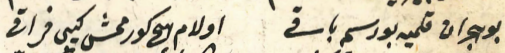

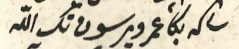

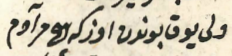

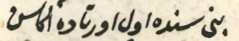

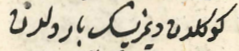

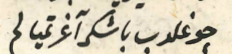

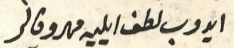

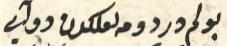

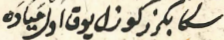

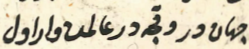

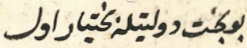

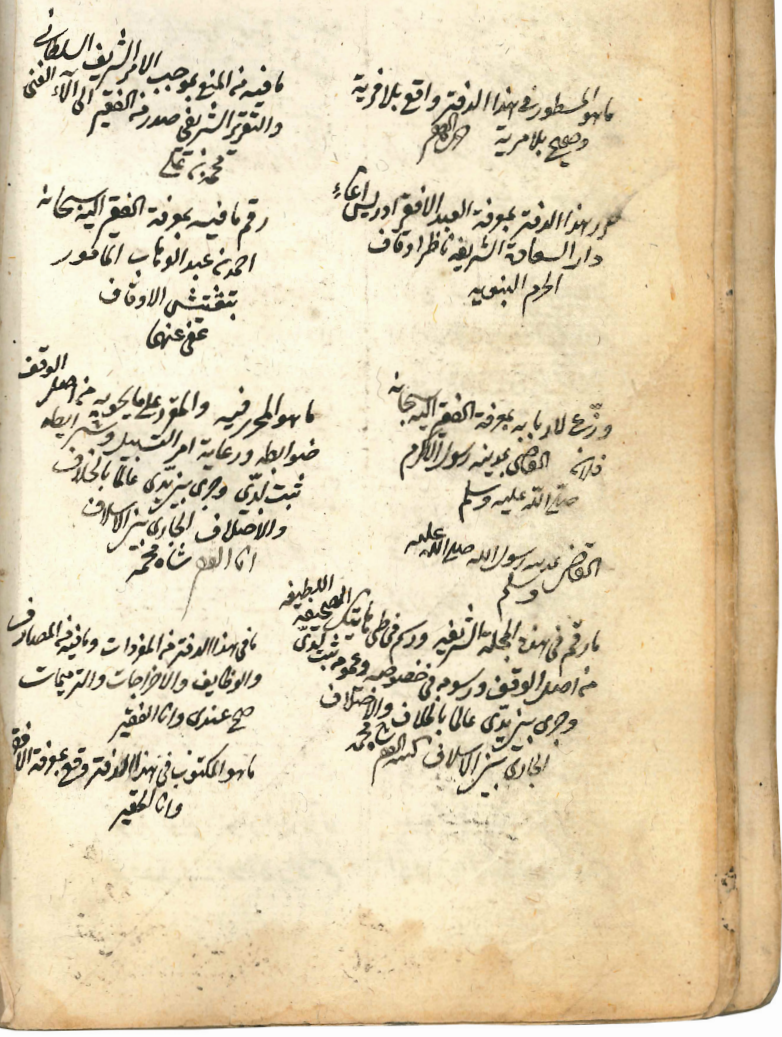

"Name-i Tacizâde Çelebi [Küsnâme]" in Mecmua-i Baldırzâde, SVIKV, IAE ŞR 525/9, 141b.

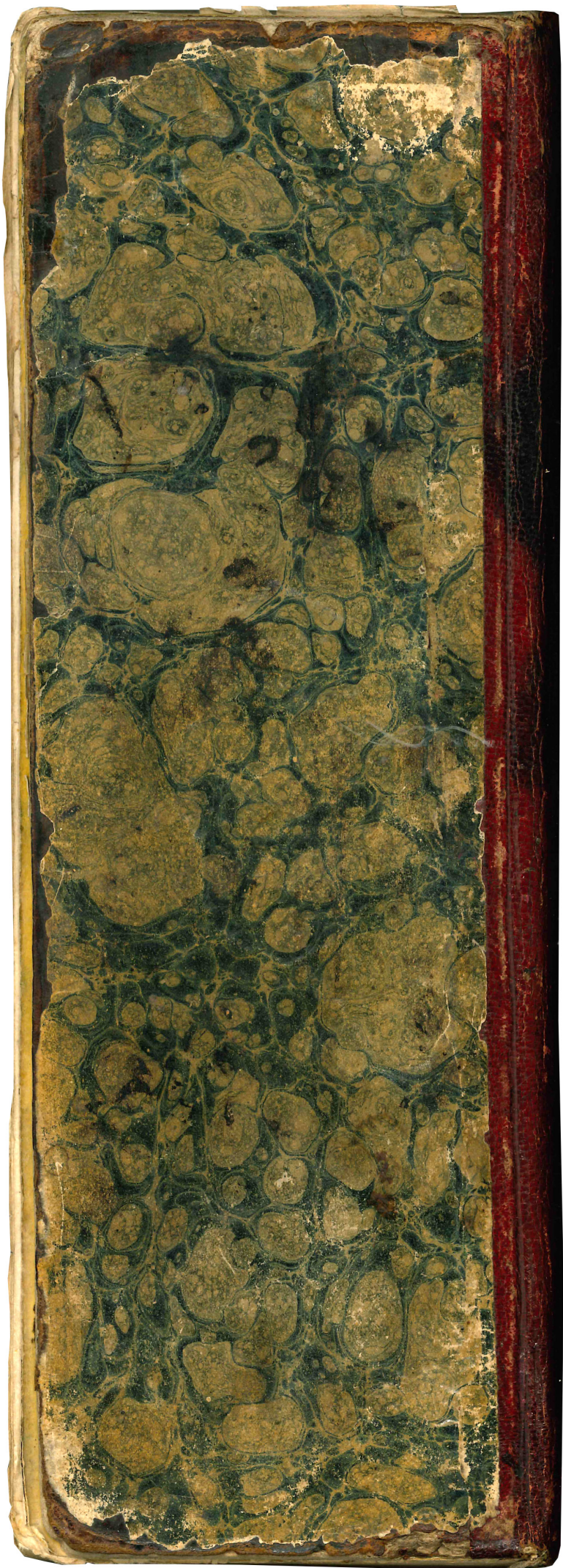

